\title{
S'appuyer sur la tradition pour renaître, innover pour résister : du vin de Tokaj aux vins de Tokaj
}

Aline Brochot

\section{OpenEdition}

\section{Journals}

Édition électronique

URL : https://journals.openedition.org/geohist/2482

DOI : 10.4000/geohist.2482

ISSN : 2264-2617

Éditeur

Association française de la Revue de géographie historique

Référence électronique

Aline Brochot, « S'appuyer sur la tradition pour renaître, innover pour résister : du vin de Tokaj aux vins de Tokaj ", Revue de géographie historique [En ligne], 19-20 | 2021, mis en ligne le 20 novembre 2021. consulté le 21 novembre 2022. URL : http://journals.openedition.org/geohist/2482 ; DOI : https:// doi.org/10.4000/geohist.2482

Ce document a été généré automatiquement le 21 novembre 2022.

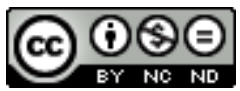

Creative Commons - Attribution - Pas d'Utilisation Commerciale - Pas de Modification 4.0 International - CC BY-NC-ND 4.0

https://creativecommons.org/licenses/by-nc-nd/4.0/ 


\title{
S'appuyer sur la tradition pour renaître, innover pour résister : du vin de Tokaj aux vins de Tokaj
}

\author{
Aline Brochot
}

\section{Introduction}

1 Jusqu'à récemment, dire «vin de Tokaj» renvoyait inévitablement à l'un des plus grands vins liquoreux au monde, le fameux Tokaji Aszú. Sa typicité et la légende forgée autour de sa diffusion dans les plus hautes sphères de la société européenne depuis le début du XVII ${ }^{\text {è }}$ iècle en ont fait un des emblèmes majeurs de la Hongrie, ainsi que le rappelait encore récemment le ministre du développement rural :

«Le vin de Tokaj, c'est toute la Hongrie dans une bouteille ».

2 L'Aszún de Tokaj est en effet un vin unique par son procédé d'élaboration, qui le distingue des autres liquoreux. Les raisins botrytisés ou passerillés (illustration 1) sont " cueillis » à la main, grain par grain, pressés et assemblés à un moût en fermentation ou à un vin nouveau dans des proportions bien définies selon le degré de sucre résiduel souhaité, avant un vieillissement en barrique. 
Illustration 1 : Raisins avec grains aszús

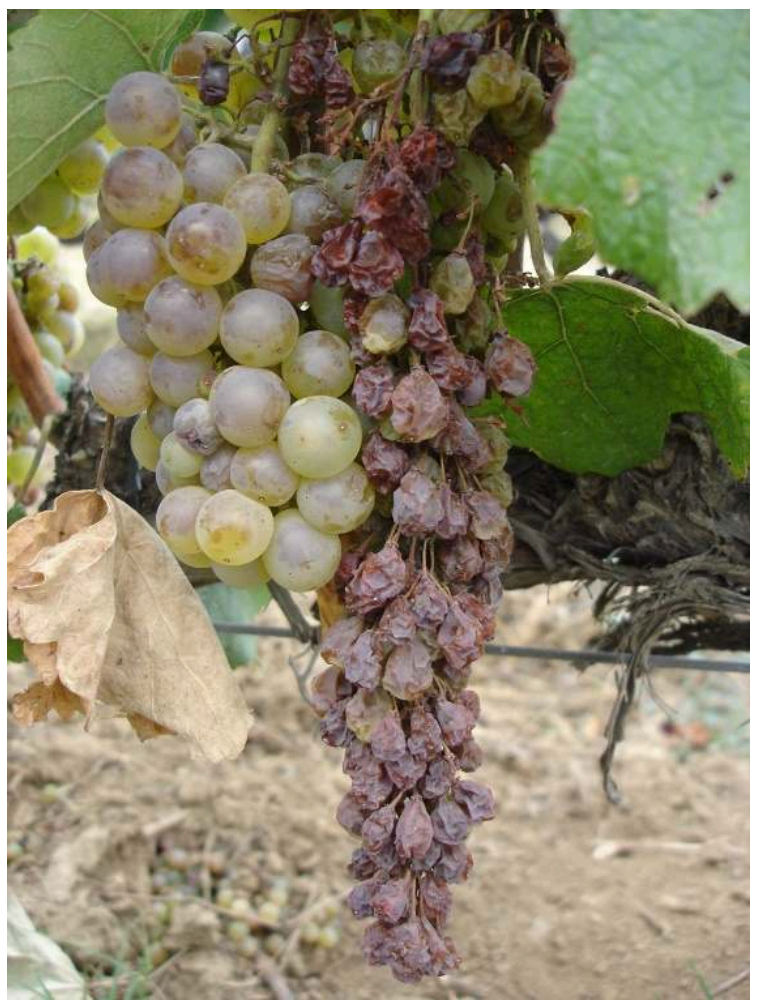

Cliché : Aline Brochot, septembre 2006

\section{La construction de la tradition du vin de Tokaj}

L'essor décisif du vignoble de Tokaj remonte à l'installation dans la région de nombreuses familles aristocratiques venues échapper à l'occupation ottomane de la partie sud de la Hongrie à partir de 1526. Sous leur impulsion, la viticulture connaît un développement considérable et les vins produits ne tardent pas à apparaitre sur les marchés nationaux et internationaux. La légende veut que ce soit la menace de la poursuite de l'invasion vers le Nord du pays qui ait repoussé les vendanges, entraînant ainsi le dessèchement des raisins et l'apparition de la pourriture noble. Les grappes pressurées telles quelles donnent alors naissance à un vin sucré, appelé fóbor ${ }^{3}$, qui connaît rapidement le succès. Peu à peu, toute l'activité viticole est centrée sur l'obtention de grains botrytisés et passerillés et l'image distinctive de la région et de son vin commence à se former. Dès 1561, une alliance de 13 communes se créée afin de fixer une première délimitation de l'aire de production exclusive de l'appellation «Tokaji» (i.e. de Tokaj). La première mention du terme "Aszú szeóleő bor » (vin passerillé) apparaît dans un glossaire de la vigne intitulé De Nomenklatura en 1570. Puis, sous l'impulsion des grands propriétaires et des négociants des villes-marchés, plusieurs règlements voient le jour à partir de 1613, fixant des normes de production et de vinification et favorisant la production des vins Aszú : règlementation de la collecte des grains Aszú ; exemption de taxes sur les grains Aszú, ce qui a pour effet de faire passer le föbor, traditionnellement fabriqué à partir de grappes entières, au second plan. Toute l'activité est désormais consacrée à la production de blancs doux et liquoreux, l'Aszú représentant le haut de la gamme, désormais consommé par les élites 
européennes ${ }^{4}$, qui en ont fait, à l'égal du champagne, un vin de prestige et de dégustation. Un tiers de la production est alors exporté, principalement vers la Pologne et la Russie ${ }^{5}$. C'est l'âge d'or de Tokaj qui va durer jusqu'à l'orée du XXè siècle.

Le prestige du vin de Tokaj est tel qu'il est aussi utilisé à des fins politiques. Ainsi, le prince Ferenc Rákóczi II, propriétaire des trois quarts du vignoble au tournant du

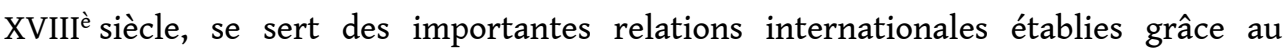
commerce de ses vins, pour demander le soutien des grandes cours européennes dans la lutte d'indépendance qu'il mène contre les Habsbourg (1703-1711). C'est, dit-on, lors d'une visite auprès de Louis XIV que ce dernier aurait nommé le vin de Tokaj, «vinum regum, rex vinorum ", soit « le roi des vins, et le vin des rois ». Le succès entraînant, ici comme ailleurs, les contrefaçons, des mesures de protection ne tardent pas à être prises. En 1736, un Comité est créé afin de lutter contre les falsifications et réglementer la production des vins Aszú. Puis, en 1737, c'est un décret royal qui délimite la première aire d'appellation de Tokaj-Hegyalja assurant aux producteurs des seules 28 communes désignées le monopole de la production des vins Aszú et réglementant les conditions de leur vinification et commercialisation.

La parution de l'Album de Tokaj-Hegyalja ${ }^{6}$ en 1867 illustre à propos la notoriété acquise par les vins de Tokaj et leur diffusion à l'échelle de l'Europe entière. Edité par la «Société vinicole de la Tokay-Hegyalja » dirigée par les plus grands propriétaires de l'époque et rédigé en quatre langues (hongrois, allemand, français, anglais), l'ouvrage se donne pour mission « de montrer de combien de beautés la nature a paré le pays où s'élève le trône du roi des vins » et présente un panorama complet de l'appellation, de ses terroirs, de ses villages et de ses vins.

6 Cinq types de vins y sont distingués. Ils renvoient aux étapes successives de la vinification des grains aszú (tableau 1) :

- l'Essenczia (essence) : jus obtenu directement par pressurage sous leur propre poids des grains aszú.

- l'Aszú : issu de l'assemblage de la pâte résultant du premier pressage avec un moût ou vin de base.

- le Forditás : troisième étape qui découle de l'assemblage du marc résultant de l'élaboration de l'Aszú avec un vin ordinaire avant un second pressurage.

- le Szamorodni (ex föbor) (le terme vient du slavon et veut dire « comme ça vient ») : résulte du pressurage des grappes entières dont les grains Aszú n'ont pas été prélevés.

- le Máslás (ou arrière-vin) : élaboré par contact d'un vin blanc ordinaire avec les lies des raisins Aszú ou de Szamorodni.

Tableau 1 : La gamme des vins de Tokaj aujourd'hui

\begin{tabular}{|l|l|l|}
\hline $\begin{array}{l}\text { TYPES DE } \\
\text { VINS }\end{array}$ & $\begin{array}{l}\text { Sucre } \\
\text { résiduel } \\
\text { minimum } \\
(\mathrm{g} / \mathrm{l})\end{array}$ & CARACTERISTIQUES \\
\hline Eszencia & 450 & $\begin{array}{l}\text { Jus libéré par pressurage naturel (sous leur propre poids) des grains } \\
\text { aszú }\end{array}$ \\
\hline $\begin{array}{l}\text { Tokaji Aszú } \\
6 \text { puttonyos }\end{array}$ & 150 & $\begin{array}{l}\text { Assemblage de grains aszú, récoltés grain par grain, avec un vin ou un } \\
\text { moût de base ; Elevage pendant 2 ans minimum dont 18 mois en fût de } \\
\text { chêne }\end{array}$ \\
\hline
\end{tabular}

Le nombre de puttonyos correspond à la quantité de grains Aszú

Revue de géographie historique, 19i2øegerée dans le vin de base pour atteindre le degré de sucre résiduel souhaité. A l'origine, le puttony désigne une hotte d'une contenance d'environ $27 \mathrm{~kg}$ de grains aszú, mis à macérer dans un fût traditionnel de 136 litres (fût de Gönc) 


\begin{tabular}{|l|l|l|}
\hline $\begin{array}{l}\text { Tokaji Aszú } \\
5 \text { puttonyos }\end{array}$ & 120 & \\
\hline $\begin{array}{l}\text { Édes } \\
\text { Szamorodni }\end{array}$ & 60 & $\begin{array}{l}\text { Szamorodni doux : Vin issu de grappes vendangées tardivement, en } \\
\text { partie botrytisées ; Pressurage des grappes entières ; Elevage pendant } \\
\text { au moins } 1 \text { an dont } 6 \text { mois en fût de chêne }\end{array}$ \\
\hline Fordítás & 45 & $\begin{array}{l}\text { Vin doux issu d'une deuxième macération sur grains aszú et nouveau } \\
\text { pressurage }\end{array}$ \\
\hline Máslás & 45 & Vin doux élaboré au contact des lies de vins aszú \\
\hline $\begin{array}{l}\text { Késői Szüret } \\
\text { édes }\end{array}$ & 45 & Vendanges tardives ; élevage en barrique \\
\hline $\begin{array}{l}\text { Késői Szüret } \\
\text { felédes }\end{array}$ & 19 & Vendanges tardives ; Semi-sucré \\
\hline Félszáraz \\
Borok
\end{tabular}

7 L'image du vignoble de Tokaj comme exclusivement producteur de vins blancs liquoreux est fixée. Elle sera reprise telle quelle jusqu'à nos jours.

\section{Les ruptures du XXè siècle}

8 Cet âge d'or va être interrompu par une série de ruptures qui vont avoir des conséquences dramatiques non seulement sur la structure du vignoble mais aussi sur la qualité des vins.

9 C'est d'abord le phylloxéra qui atteint la région en 1886 et détruit en une dizaine d'années près de $90 \%$ des vignes. Les replantations se font en majorité avec les deux cépages blancs déjà majoritaires : le Furmint et le Hárslevelü7. Cependant, ce sont les bouleversements occasionnés par les deux guerres mondiales qui ont les effets les plus destructeurs. Les mesures issues du Traité de Trianon de 1920 se révèlent dévastatrices pour cette région, entraînant une triple rupture, à la fois géopolitique, économique et commerciale. En effet, la Hongrie se voit amputée des trois quarts de son territoire et la région de Tokaj devient zone frontière, trois communes de l'appellation étant en outre transférées sous souveraineté slovaque. Par suite, les routes commerciales traditionnelles, notamment avec la Pologne, plaque tournante des échanges depuis le 
XVIè siècle, sont coupées accentuant la désorganisation des structures de production et la perte de débouchés.

10 Les suites de la deuxième guerre amplifient encore la fracture avec le reste de l'Europe occidentale. La Hongrie passée dans l'orbite soviétique à la fin du conflit, le vignoble fait face à une politique de collectivisation qui affecte en profondeur les structures et les choix de production. Les grands domaines historiques de l'aristocratie et de la haute bourgeoisie, les seuls ayant une taille économique suffisante pour être présents sur les marchés internationaux, sont nationalisés à partir de 1948 (1400 hectares sur les 7000 hectares plantés) et intégrés à partir de 1952 au sein d'une ferme d'Etat, le Borkombinat, qui détient le monopole de la vinification et de la commercialisation . $^{8}$ S'opère alors une nouvelle rupture, qualitative cette fois, avec une très forte réduction de la part des vins Aszú et l'orientation vers une production de masse de vins doux de faible qualité (des Szamorodni expédiés en vrac), principalement à destination du marché russe devenu le principal débouché, dévalorisant par là-même l'image de produit de luxe acquise par les vins de Tokaj.

11 A la fin des années 1980, le contexte économique s'est profondément dégradé, le Borkombinat est en quasi-faillite et le vin de Tokaj a presque totalement disparu non seulement des marchés européens, mais aussi de la mémoire des consommateurs qui en ont oublié le goût et l'image.

Il faut attendre la toute fin du XXè siècle pour que le nom de Tokaj réapparaisse sur le marché international du vin sous l'impulsion de grands noms de la vitiviniculture locale et de quelques investisseurs étrangers. Tous désireux de renouer avec l'âge d'or, ils enclenchent une dynamique de reconstruction tant matérielle que symbolique en s'appuyant sur ce qui a fait les grandes heures de Tokaj : la tradition de l'Aszú.

\section{S'appuyer sur la tradition pour renaître}

13 Avec la disparition du "bloc de l'Est ", s'ouvre une nouvelle ère pour le vignoble de Tokaj. Parmi les premières mesures gouvernementales figurent la privatisation de l'économie et le démantèlement du domaine d'Etat dès 1990.

L'acte fondateur de cette « renaissance » est le démantèlement du Borkombinat, dont les opérations sont confiées à son dernier directeur œnologue. Celui-ci ambitionne de recréer les structures qui ont fait de Tokaj un vignoble d'excellence, réputé pour la qualité exceptionnelle de ses vins. Et c'est notamment sur la base de l'Album de Tokaj qu'il va s'appuyer pour redessiner les contours de grands domaines historiques sur les 1400 hectares confisqués au sortir de la deuxième guerre mondiale. Cinq entités comprenant vignes, caves et stocks - correspondant à d'anciens domaines royaux et impériaux, sont ainsi créées puis proposées à des partenaires étrangers sous la forme de sociétés conjointes. Les candidats retenus sont des sociétés viticoles déjà présentes sur le marché international du vin. Quatre sont françaises, filiales de compagnies d'assurances déjà très investies dans le monde viticole, en particulier bordelais, la cinquième dépend d'un grand groupe viticole espagnol ${ }^{9}$. L'Etat conserve néanmoins une structure propre comprenant vignes, installations de vinification et de stockage.

Parallèlement, d'autres investisseurs ou des grands noms de la vitiviniculture internationale attirés par la réputation de Tokaj (britanniques, américains, japonais, français...) reprennent d'anciennes coopératives ou s'associent avec des viticulteurs du 
cru pour créer de nouveaux domaines. De leur côté, quelques descendants d'anciens grands propriétaires dont les biens avaient été confisqués reconstituent des propriétés familiales par rachat de vignes au moyen de bons de privatisation distribués par l'Etat.

Quels que soient leur statut ou origine, tous se donnent pour mission de faire revivre les grands vins de Tokaj et de les faire (re)connaître à l'étranger, à l'instar de ce grand domaine créé en 1990 :

«Our goal is to make wines which can stand alongside the finest in the world and to return the wines of Tokaj to international acclaim. $»^{10}$.

Il $\mathrm{y}$ a, partout et pour tous, la volonté de reprendre l'histoire là où le régime collectiviste l'avait effacée. Les symboles royaux: couronnes, blasons, la formule " vinum regum, rex vinorum » reprennent leur place sur les étiquettes de bouteilles et sur les supports de communication (illustration 2).

Les structures professionnelles antérieures qui avaient été dissoutes lors de la collectivisation sont reconstituées. Ainsi, en 1994 est créée par décret l'« Organisation des communes de montagne ", interprofession organisée par commune et par région viticole, qui reprend une dénomination ancienne. Puis, en 1995, est créée «l'Union des grands crus de Tokaj» qui rassemble à l'image de l'ancienne "Société vinicole de la Tokay-Hegyalja " les plus grands domaines. Plus communément appelée «Tokaj Renaissance ", elle va, de façon symbolique rééditer l'Album de Tokaj en 2001, qui fait plus que jamais figure de référence absolue et dont les gravures sont affichées dans tous les lieux d'accueil, caves, hôtels, restaurants ... comme autant de rappels ou de preuves de la gloire passée.

Illustration 2 : Logo de l'association Tokaj Renaissance qui reprend la formule « vinum regum rex vinorum "
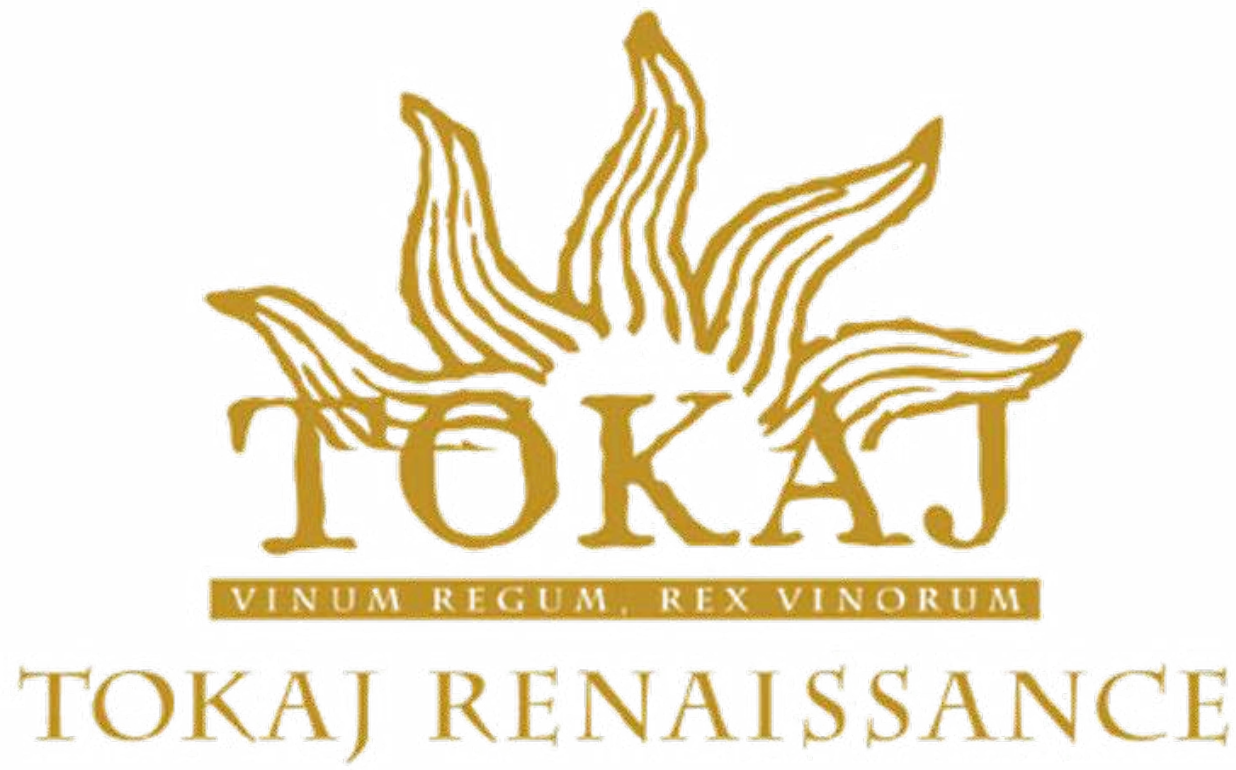

Source : site tokaji.hu

Dans cette entreprise de restauration, l'Aszú est bien sûr au centre de toutes les préoccupations et de tous les projets. Dès leur installation, les principaux investisseurs 
en font le centre de leur gamme et l'ambassadeur incontournable des vins de Tokaj dans la reconquête des marchés à l'export.

Cependant, c'est sur des bases technologiques nouvelles que la production est lancée, inaugurant une période de débats animés autour du respect de la tradition (Brochot, Plet, 1999). Partisans de la méthode dite traditionnelle avec élevage long en fûts et promoteurs de l'élaboration en cuves s'opposent un temps, avant que la technologie importée par les investisseurs finisse de s'imposer devant les bons résultats obtenus. L'année 1993 est ainsi saluée comme le début d'une nouvelle ère avec la mise sur le marché des premiers Aszús "nouveau style ».

Durant toute la décennie, s'élabore ainsi la «nouvelle » tradition de Tokaj, basée sur la production de vins d'élite, élaborés certes selon des méthodes modernes, mais dont on façonne et diffuse une image fidèle à la légende du «vin d'or ». Régulièrement primés dans les grands concours internationaux, ils reprennent leur place au sommet de la hiérarchie des liquoreux, notamment auprès d'un public d'amateurs à la recherche de vins historiques.

Pourtant, cette incontestable réussite peine à masquer les prémices d'une nouvelle crise que l'image glorieuse de Tokaj ne parvient pas à réduire.

\section{Innover pour « résister »}

Les années 2000 marquent un tournant à plusieurs égards. En effet, si le nom de Tokaj a retrouvé une certaine reconnaissance dans les milieux internationaux du vin grâce à la réactivation des figures et symboles de son glorieux passé, d'autres facteurs interviennent alors pour limiter leur reconquête du marché, intérieur comme à l'export.

24 L'inscription en 2002 de la "région viticole historique de Tokaj " sur la Liste du Patrimoine mondial de l'UNESCO a sans nul doute représenté une forme de reconnaissance ultime de la valeur historique et patrimoniale de la région, et par extension de ses vins. Il s'agissait pour l'Etat hongrois de relancer, par l'intermédiaire du tourisme, l'économie de la région mise à mal par la désindustrialisation. Pourtant, cet espoir est en grande partie déçu, la région ne répondant pas aux critères nécessaires pour un réel développement œnotouristique (région-frontière; peu d'infrastructures d'accueil ; loi très restrictive sur l'alcool au volant...).

Les transformations les plus déterminantes, aussi bien sur le plan de la production que sur celui du paysage, interviennent à la suite de l'entrée de la Hongrie dans l'Union Européenne en 2004 grâce à l'afflux de subventions qui favorisent une nouvelle vague d'investissements, en majorité de la part de Hongrois attirés par le prestige de Tokaj. Si cela débouche sur l'apparition de plusieurs nouveaux domaines (carte 1), ainsi que sur la transformation rapide de certains coteaux et villages, cela a aussi pour effet d'accroître la concurrence interne entre les différents producteurs et d'amplifier une tendance récurrente à la surproduction. D'autant plus, et c'est peut-être le moteur le plus décisif de ce tournant, que les goûts des consommateurs ont changé et qu'on enregistre, en Hongrie comme ailleurs, une désaffection générale pour les vins liquoreux, encore accentuée par le positionnement dans le haut de gamme des vins Aszú avec des prix en conséquence élevés ${ }^{11}$. 
Carte 1 : La région viticole de Tokaj

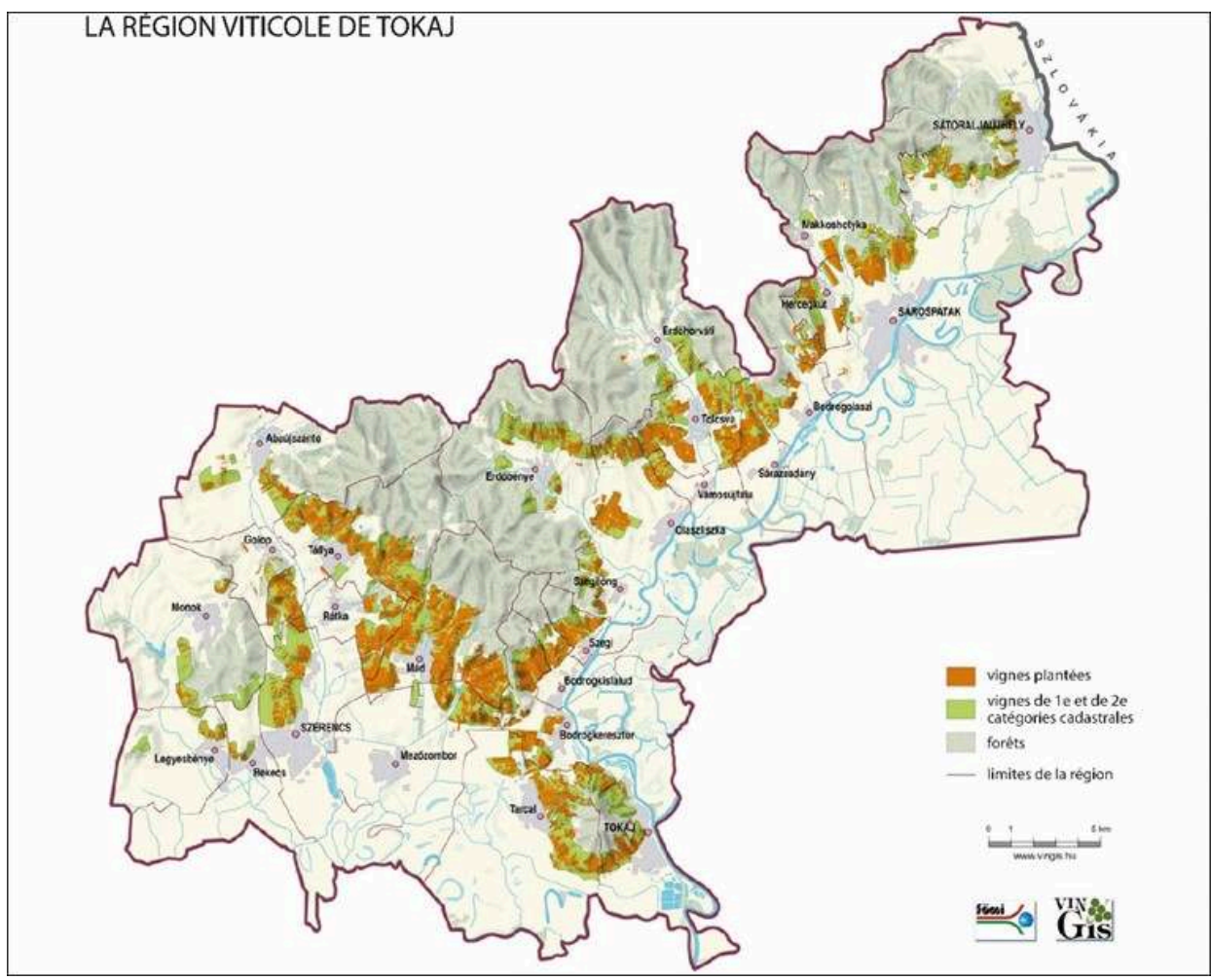

Source : FÖMI (Institut de Géodésie, Cartographie et Télédétection)

Dès lors, la recherche d'alternatives au "tout liquoreux » devient la préoccupation majeure de la plus grande partie des producteurs. Cela passe en tout premier lieu par une augmentation significative de la production de vins blancs secs à base de furmint, le cépage-roi de la région. La vinification en sec comporte en effet le double avantage de pouvoir effectuer la vendange dès la maturité des raisins (sans attendre une botrytisation toujours aléatoire) et de produire des vins commercialisables plus rapidement ${ }^{12}$, assurant par là-même des rentrées de trésorerie plus régulières. Devant le succès rencontré pour ce type de vin, "facile à boire ", pouvant accompagner tout un repas et surtout beaucoup moins chers, la pratique se généralise rapidement, au point de bientôt représenter plus du quart de la production totale dans les années 2010 et de paraître aux yeux de certains comme l'amorce d'un « nouvel âge d'or » (illustration 3). 
Illustration 3 : Un nouvel âge d'or pour Tokaj ? «Comment une révolution tranquille née à Mád peut changer notre manière de voir Tokaj »

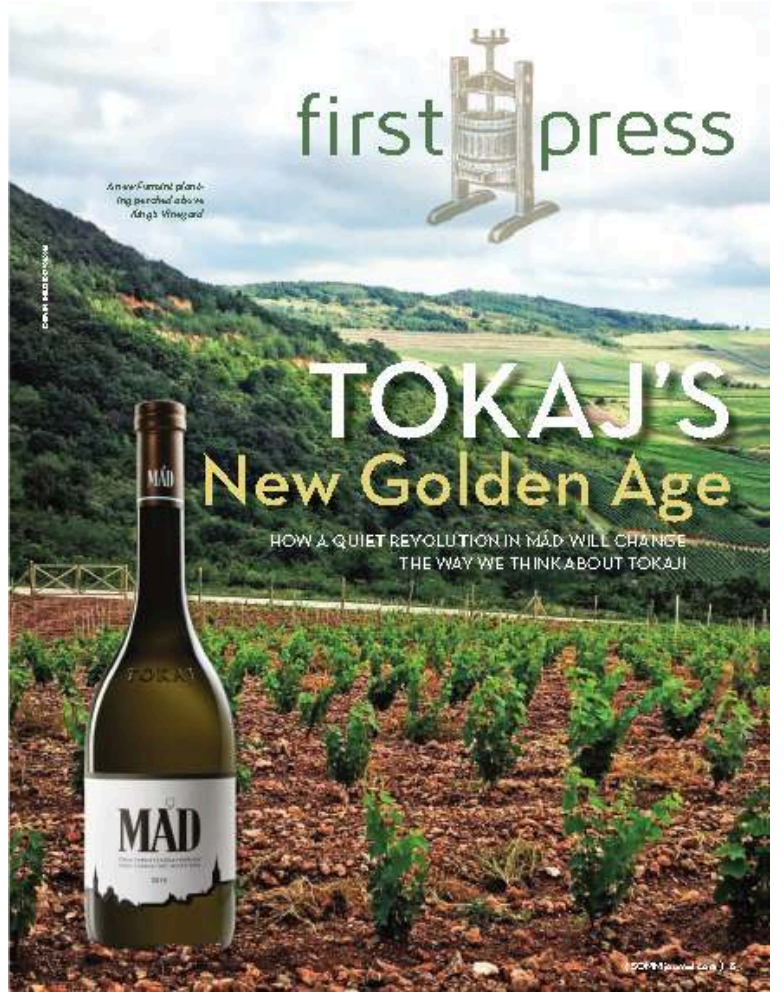

Source : The SOMM Journal, octobre 2016

Cependant, cela entraîne aussi un accroissement de la concurrence du fait de la multiplication du nombre de producteurs sur ce segment. Diverses stratégies orientées vers la «typicisation » et la montée en qualité des vins sont alors mises en œuvre, en particulier au sein des plus grosses unités, qui disposent de plus de moyens technologiques et financiers et qui peuvent se permettre de prolonger la durée d'élevage de leurs vins. L'accent est mis sur le terroir avec l'apparition de cuvées portant, sur le modèle bourguignon, le nom de la parcelle d'origine, a fortiori lorsqu'il s'agit de crus historiques réputés.

A nouveau type de vin, nouvelle présentation : Les étiquettes sont modernisées, laissant de côté les références historiques pour adopter des graphismes plus dans l'air du temps et susceptibles d'attirer un public jeune et sans réelles connaissances ou a priori sur le vin.

En outre, la nécessité de se faire (re)connaître sur les marchés à l'export incite à créer une image de marque et des dénominations plus immédiatement compréhensibles et lisibles. Ainsi, des cuvées "Late harvest", plus connues à l'international, font leur entrée dans la gamme des vins proposés. Certains vont même jusqu'à simplifier à l'extrême les dénominations en proposant simplement du «sweet Tokaj» et du «dry Tokaj ». Outre qu'il s'agit là d'adopter un mode de communication qui réponde aux standards du commerce international, toutes ces nouvelles dénominations traduisent la volonté de se positionner durablement sur le marché anglo-saxon, en particulier britannique qui connaît une progression spectaculaire et où le furmint fait l'objet d'un véritable engouement ${ }^{13}$. 
Si les liquoreux perdent globalement du terrain en termes de volumes, ils n'en demeurent pas moins au cœur des préoccupations pour l'avenir de l'appellation. Pour la plupart des producteurs et acteurs de la filière, l'Aszú est et doit rester le vin-phare de Tokaj. De nouvelles règles sont édictées en $2013^{14}$ afin de garantir une qualité optimum et d'apporter plus de lisibilité sur les marchés internationaux. Le cahier des charges prévoit notamment de relever le taux minimum de sucre résiduel pour pouvoir prétendre à la dénomination «Aszú » (tableau 2), ce qui a pour effet de supprimer les Aszú faiblement sucrés (3 et 4 puttonyos). La mention du nombre de puttonyos elle-même n'est plus obligatoire et laissée à la libre appréciation du producteur, en fonction de ses choix de marketing.

Tableau 2 : Nouvelles spécifications pour les vins Aszú

\begin{tabular}{|c|c|c|c|c|c|c|}
\hline \multirow{2}{*}{\begin{tabular}{|l} 
\\
$\begin{array}{l}\text { Mesures } \\
\text { traditionnelles }\end{array}$
\end{tabular}} & \multicolumn{3}{|c|}{ SPECIFICATIONS AVANT 2013} & \multicolumn{3}{|c|}{$\begin{array}{l}\text { SPECIFICATIONS APRES } 2013 \\
\text { (L'indication du nom et du nombre de } \\
\text { puttonyos n'est plus obligatoire) }\end{array}$} \\
\hline & $\begin{array}{l}\text { Sucre } \\
\text { résiduel } \\
\text { minimum } \\
(\mathrm{g} / \mathrm{L})\end{array}$ & $\begin{array}{l}\text { Alcool } \\
\text { minimum } \\
(\%)\end{array}$ & $\begin{array}{l}\text { Vieillissement } \\
\text { minimum, } \\
\text { années (dont en } \\
\text { fût) }\end{array}$ & $\begin{array}{l}\text { Sucre } \\
\text { résiduel } \\
\text { minimum } \\
(\mathrm{g} / \mathrm{L})\end{array}$ & $\begin{array}{l}\text { Alcool } \\
\text { minimum } \\
(\%)\end{array}$ & $\begin{array}{l}\text { Vieillissement } \\
\text { Minimum, } \\
\text { années (dont en } \\
\text { fût) }\end{array}$ \\
\hline $\begin{array}{ll}\text { Aszú } & 3 \\
\text { puttonyos }\end{array}$ & 60 & $9 \%$ & $3(2)$ & & & \\
\hline $\begin{array}{ll}\text { Aszú } & 4 \\
\text { puttonyos }\end{array}$ & 90 & $9 \%$ & $3(2)$ & & & \\
\hline $\begin{array}{ll}\text { Aszú } & 5 \\
\text { puttonyos }\end{array}$ & 120 & $9 \%$ & $3(2)$ & 120 & $9 \%$ & $2(1,5)$ \\
\hline $\begin{array}{ll}\text { Aszú } & 6 \\
\text { puttonyos } & \end{array}$ & 150 & $9 \%$ & $3(2)$ & 150 & $9 \%$ & $2(1,5)$ \\
\hline Eszencia & $>450$ & $1,2 \%$ & $3(2)$ & $>450$ & $1,2 \%$ & \\
\hline
\end{tabular}

31 Puis, en 2016, une nouvelle série de mesures porte sur les autres types de vins doux traditionnels (szamorodni, forditás, máslás). Le degré d'alcool minimum est modifié, la durée de vieillissement est réduite pour permettre une mise sur le marché plus précoce, les normes d'étiquetage sont révisées. Toutes ces mesures visent aussi à donner plus de liberté aux viticulteurs dans la « stylisation » de leurs vins d'une part et à simplifier l'image des vins de Tokaj, quelque peu confuse pour des consommateurs non-initiés d'autre part. Dans cette optique, beaucoup optent alors pour la réduction de leur gamme, ne présentant plus qu'un vin de chaque catégorie: un Furmint sec; un Vendanges tardives ; un Aszú ; un Eszencia, le cas échéant.

Les années 2010 sont par ailleurs marquées par un autre phénomène, totalement inédit pour Tokaj : l'émergence de vins effervescents. Dès 2011, deux producteurs à la tête 
d'exploitations relativement importantes commencent à élaborer du Pezgö, bien que ce type de vins ne soit pas encore reconnu. Pourtant, ce nouveau produit remporte rapidement le succès auprès des consommateurs hongrois, en particulier les plus jeunes pour lesquels il représente une alternative bon marché au champagne et autres mousseux d'importation. Représentant une opportunité bienvenue en termes de diversification, beaucoup suivent cette voie et, appuyés par le Conseil des Communes de montagne, parviennent à convaincre les autorités de modifier la loi en 2017. Ce mouvement est même désormais largement accompagné par l'Etat, via la mise en service en 2019 de trois nouvelles unités coopératives, intégralement financées sur des fonds publics. Destinées avant tout à aider les petits producteurs qui ne disposent pas des infrastructures nécessaires, elles proposent la vinification et l'embouteillage de tous types de vin, y compris une chaîne de production complète de vins effervescents (avec notamment une batterie de gyropalettes).

33 Enfin, et comme partout ailleurs, le réchauffement climatique apporte aussi son lot d'incertitudes et la nécessité d'élaborer des stratégies nouvelles. Quelques grands domaines ont d'ores et déjà expérimenté sur quelques parcelles la production de cépages rouges. Pinot noir, Merlot, Syrah font ainsi figure de débouchés possibles, quitte à se placer sur un marché international certes plus concurrentiel, mais jugé plus ouvert. De leur côté, quelques producteurs indépendants, s'appuyant sur le fait que des cépages rouges étaient produits à Tokaj dans la période pré-phylloxérique, ont lancé une production régulière, qui si elle participe davantage d'une stratégie de distinction n'en représente pas moins une voie possible de diversification et un objet de curiosité si ce n'est d'intérêt pour des consommateurs à la recherche de produits originaux.

\section{Conclusion : Et demain, de quoi Tokaj sera-t-il le nom ?}

34 Ainsi, en une vingtaine d'années, la région viticole de Tokaj a vu émerger des produits nouveaux, certains totalement inédits et rompant avec la tradition des grands liquoreux de Tokaj et qui, pour être en accord avec l'évolution du contexte économique et de la demande des consommateurs, n'en soulèvent pas moins de nombreuses interrogations pour l'avenir.

L'élargissement de la gamme des vins a déjà eu pour effet la diminution corrélative de la part des vins Aszú dans la production totale. Dans les années 2010, l'appellation commercialise 20 à $25 \%$ de blanc sec et seulement 5 à $8 \%$ de vins Aszú. Et, dans un audit réalisé en 2015 à la demande du Conseil de Développement de la région viticole de Tokaj, on estime à 60-65\% la part des blancs secs à la fin de la décennie 2020 ! C'est donc à une véritable mutation que l'on assiste (Brochot, Albert, 2020), presque un changement de paradigme.

Alors, qu'en sera-t-il demain de la tradition de Tokaj? Est-ce que le nom de Tokaj continuera à rimer avec Aszù ? Les évolutions constatées aujourd'hui ne risquent-elles pas de faire perdre sa spécificité et son pouvoir symbolique à ce vignoble en en faisant finalement une région viticole « comme les autres»? 


\section{BIBLIOGRAPHIE}

Brochot A., Plet F., 1999, « Le choc des investissements étrangers dans le vignoble de Tokaj

(Hongrie) », dans Gouëset V., Investissements étrangers et milieu local, Rennes, Presses

Universitaires de Rennes, p. 289-309.

Brochot A., Albert K., 2020, « De transition en mutation, le vignoble de Tokaj aujourd'hui », POUR, $\mathrm{n}^{\circ} 237-238, \mathrm{p} .103-115$.

Havens C., 2016 « Tokaj's new golden age ", The SOMM Journal, october/november vol. 3, n 5, p.

5-7.

Szabo J., Török E., 1867, Album de Tokaj-Hegyalja, Pest, (reprint : 2001).

\section{NOTES}

1. Press Office of the Ministry of Rural Development, octobre 2011. Tokaj est aussi évoqué dans l'hymne national hongrois : «Et tu permis que du nectar de Tokaj nos coupes soient pleines ».

2. Littéralement Aszú signifie « desséché » ou « séché au soleil ».

3. Le fóbor (ou vin principal) peut être considéré comme l'ancêtre du szamorodni actuel.

4. Les cours de Russie, de Pologne, d'Autriche, de France et d'Angleterre sont les principaux clients.

5. En 1733, un « comité russe du commerce du vin » est créé pour fournir la cour du tsar. Plus de 100 fûts de vin de Tokaj sont expédiés chaque année jusqu'en 1798.

6. Préface de l'Album de Tokaj-Hegyalja, Société vinicole de la Tokay-Hegyalj, 1867, p. 3.

7. Les cépages autorisés par l'Appellation sont le Furmint (60\% des superficies plantées), Hárslevelű (30\%), Sárgamuskotály (Muscat blanc à petits grains) (5\%), Zéta (4\%), Kövérszőlő et $\operatorname{Kabar}(<1 \%)$.

8. Même si de nombreuses micro-propriétés, jardins familiaux de quelques ares, subsistent parallèlement (elles assurent entre 60 et $80 \%$ de la production), elles ne peuvent prétendre alimenter le marché à l'export. La production est essentiellement destinée à l'autoconsommation ou à la fourniture de raisin à l'entreprise d'Etat.

9. GAM Audy (Megyer et Pajzos) en 1991 ; Axa Millésimes (Disznókő) en 1992 ; Grands Millésimes de France (Hétszőlő) en 1992 ; Bodegas Vega Sicilia (Oremus) en 1993.

10. Royal Tokaji Wine Company : https://royal-tokaji.com/

11. Par exemple, le prix d'une bouteille d'Aszú 5 puttonyos de $50 \mathrm{cl}$ oscille entre 35 et 60 euros et beaucoup plus selon le millésime.

12. Le vin blanc sec (Fehér bor), élevé en barriques neuves durant 10 mois, est commercialisable un an après la vendange.

13. " Justin Keay on why the UK wine trade is going Mád for Furmint ", The buyer, february 3, 2019.

14. «Tokaj product specifications ». Règlement adopté par le Conseil des Communes de montagne de la région de Tokaj, organisme de gestion et de certification proche de l'INAO français. 


\section{RÉSUMÉS}

Jusqu'à l'orée du XXI ̀̀ siècle, évoquer le nom de Tokaj renvoyait essentiellement à l'un des plus grands vins liquoreux, réputé dans toute l'Europe depuis le XVII è siècle et devenu l'un des produits les plus emblématiques de la Hongrie. Bien que la région de Tokaj ait connu de nombreux bouleversements au cours du temps, la typicité des vins produits dans l'appellation était demeurée intacte grâce à la perpétuation d'une tradition de vinification bien établie et dûment codifiée de son produit-phare, l'Aszú.

C'est sur cette tradition que les acteurs de la « renaissance » du vignoble de Tokaj se sont appuyés après la privatisation des années 1990 pour restaurer à la fois les structures de production et la réputation internationale du vin de Tokaj, mises à mal durant la période collectiviste.

Pourtant, et malgré l'incontestable succès de ce renouveau, les évolutions rapides des contextes économiques et sociétaux à l'échelle nationale comme internationale, ont graduellement poussé les producteurs à explorer des voies inédites pour la région et à développer une nouvelle gamme de produits qui leur permet de maintenir le niveau et la rentabilité de leur exploitation.

C'est ce passage d'une tradition revendiquée comme gage de retour vers l'âge d'or à une diversification/innovation plus ou moins contrainte par l'évolution du marché que nous proposons d'exposer. Comment, du « vin de Tokaj », produit unique et patrimonial, est-on passé aux « vins de Tokaj », multiples et répondant aux impératifs économiques du moment?

Until the beginning of the 21st century, the name of Tokaj essentially referred to one of the greatest sweet wines, renowned throughout Europe since the 17th century and one of the most emblematic products of Hungary. Although the Tokaj region has undergone many upheavals over the years, the typicality of the wines produced in the appellation has remained intact thanks to the perpetuation of a well-established and duly codified winemaking tradition of its iconic product, Aszú wine.

It is on this tradition that the actors of the "renaissance" of the Tokaj vineyard relied after the privatisation of the 1990s to restore both the production structures and the international reputation of Tokaj wine, which were damaged during the collectivist period.

However, despite the undeniable success of this revival, the rapid changes in the economic and societal contexts, both nationally and internationally, have gradually pushed producers to explore new ways for the region and to develop a new range of products that enable them to maintain the level and profitability of their estates.

It is this passage from a tradition claimed as a guarantee of a return to the golden age to a diversification/innovation more or less constrained by the evolution of the market that we propose to expose. How, from "Tokaj wine", a unique and patrimonial product, have we moved on to "Tokaj wines", multiple and responding to the economic imperatives of the moment?

\section{INDEX}

Keywords : Tokaj, Tokaj wines, Aszú, tradition, diversification, marketing

Mots-clés : Tokaj, vins de Tokaj, Aszú, tradition, diversification, marché 
AUTEUR

ALINE BROCHOT

Ingénieur de recherche au CNRS

UMR 7533 - LADYSS

aline.brochot@univ-paris1.fr 